\title{
Universal homodyne tomography with a single local oscillator
}

\author{
G. Mauro D'Ariano and Massimiliano F. Sacchi \\ Dipartimento di Fisica "A. Volta", Università di Pavia and INFM, \\ via A. Bassi 6, I-27100 Italy \\ Prem Kumar \\ Department of Electrical and Computer Engineering, Northwestern University, \\ Evanston, IL 60208, USA
}

(April 7, 2018)

\begin{abstract}
We propose a general method for measuring an arbitrary observable of a multimode electromagnetic field using homodyne detection with a single local oscillator. In this method the local oscillator scans over all possible linear combinations of the modes. The case of two modes is analyzed in detail and the feasibility of the measurement is studied on the basis of Monte-Carlo simulations. We also provide an application of this method in tomographic testing of the GHZ state.
\end{abstract}




\section{INTRODUCTION}

Optical homodyne tomography is a well-established quantitative method for measuring the quantum state of radiation and for obtaining the expectation value of arbitrary observables of the field [1] [3] (for a review see Ref. [4]). The success of optical homodyne tomography has stimulated research relating to the state-reconstruction procedures in other fields, such as in the realm of atomic [5], molecular [6], and ion-trap [7] physics. As a matter of fact, the tomographic method is a kind of universal detection technique [8], with which one can measure any observable $O$ of the field by averaging a suitable unbiased estimator $\mathcal{E}[O](x, \phi)$ over the homodyne data $x$ at random phase values $\phi$. Single-mode homodyne tomography can be immediately generalized to multimode fields. For factorized multimode operators $O=O_{1} \otimes O_{2} \otimes \ldots \otimes O_{n}$ the corresponding estimator is just the product of the estimators for each of the single-mode operators $O_{1}, O_{1}, \ldots, O_{n}$. By linearity the estimator can then be extended to generic multimode operators. However, such a simple generalization requires a separate homodyne measurement for each of the modes, which cannot be achieved in practice when the modes of the field are not spatio-temporally separated. For this reason, tomographic methods have been devised which either use only a single local oscillator (LO) [9], or avoid the use of conventional homodyne detection [10]. However, both the methods work for only two modes of the field, and the self-homodyne method of Ref. [10] is suitable only in special experimental situations (e.g., in the tomography of parametrically downconverted radiation). Therefore, a more general multimode tomographic method is needed, especially in consideration of the possibility of a precise analysis for pulsed fields, for which the problem of mode matching between the LO and the detected fields (determined by their relative spatio-temporal overlap) [11] gives a detrimental contribution to the overall quantum efficiency.

In this paper we propose a general method for measuring an arbitrary observable of the multimode electromagnetic field, which uses homodyne detection with a single LO. We provide the rule for evaluating the "unbiased estimator" of a generic multimode operator. The quantum expectation value of the operator can then be obtained for any unknown state of the radiation field through an average of this estimator over the homodyne outcomes that are collected using a single LO which scans over different linear combinations of the incident modes. The paper is organized as follows: In Sec. II we present the general method for obtaining the estimator pertaining to an arbitrary multimode operator. Upon averaging this estimator over the homodyne outcomes, one obtains the quantum expectation value of the corresponding operator. We specialize to observables corresponding to the matrix elements of the multimode density operator and to the total number of photons. In the twomode case we explicitly derive the estimator for the four-dimensional Q-function and for the moments generating function of the total number of photons. In Sec. III we investigate the experimental conditions for extracting the joint photon-number probability and the distribution of the total number of photons for two-mode quantum states. We present the results of some Monte-Carlo simulations for the twin-beam state that is produced by nondegenerate parametric amplification (spontaneous downconversion). We average the estimators obtained in Sec. II over the homodyne data that are distributed according to the theoretical homodyne probability evaluated in the Appendix. The simulations show

that the measurement is feasible for quantum efficiency values of the homodyne detector 
in the $80-90 \%$ range and with the number of experimental data samples of order $10^{6}-10^{7}$. In Sec. IV we show an application of our method in measurement of the three-particle maximally-entangled state called the GHZ state. In such a case the number of radiation modes is six and a more suitable arrangement of the tomographic machine requires the use of three LO's. The results of Monte-Carlo simulations show that for homodyne detectors with quantum efficiency value $\eta=85 \%$ one needs about $10^{7}$ data samples to reconstruct the state with a relatively small statistical error. Finally, some conclusions are drawn in Sec. V.

\section{THE GENERAL METHOD}

For a single-mode radiation field one has the following resolution of the identity on the Hilbert-Schmidt space:

$$
O=\int \frac{\mathrm{d}^{2} z}{\pi} \operatorname{Tr}\left[O D^{\dagger}(z)\right] D(z),
$$

where $O$ is a Hilbert-Schmidt operator, the integral is extended to the complex plane $\mathbb{C}$ for $z$, and $D(z)=\exp \left(z a^{\dagger}-z^{*} a\right)$ denotes the displacement operator for the field mode with annihilation and creation operators $a$ and $a^{\dagger}$, respectively, having the commutation relation $\left[a, a^{\dagger}\right]=1$. Equation (1) simply follows from the orthogonality relation for displacement operators $\operatorname{Tr}\left[D(z) D^{\dagger}\left(z^{\prime}\right)\right]=\delta_{2}\left(z-z^{\prime}\right), \delta_{2}(z)$ denoting the Dirac delta-function on the complex plane. Equation (1) is the starting point of our method; it can be easily generalized to any number of modes as follows:

$$
\begin{aligned}
O & =\int \frac{\mathrm{d}^{2} z_{0}}{\pi} \int \frac{\mathrm{d}^{2} z_{1}}{\pi} \ldots \int \frac{\mathrm{d}^{2} z_{M}}{\pi} \operatorname{Tr}\left\{O \exp \left[\sum_{l=0}^{M}\left(-z_{l} a_{l}^{\dagger}+z_{l}^{*} a_{l}\right)\right]\right\} \\
& \times \exp \left[\sum_{l=0}^{M}\left(z_{l} a_{l}^{\dagger}-z_{l}^{*} a_{l}\right)\right],
\end{aligned}
$$

where $a_{l}$ and $a_{l}^{\dagger}$, with $l=0, \ldots, M$ and $\left[a_{l}, a_{l^{\prime}}^{\dagger}\right]=\delta_{l l^{\prime}}$, are the annihilation and creation operators, respectively, of the $M+1$ independent modes, and $O$ now denotes an operator over all the modes. Using the following hyper-spherical parameterization for $z_{l} \in \mathbb{C}$

$$
\begin{aligned}
z_{0}=\frac{i}{2} k u_{0}(\vec{\theta}) e^{i \psi_{0}} & \doteq \frac{i}{2} k e^{i \psi_{0}} \cos \theta_{1} \\
z_{1}=\frac{i}{2} k u_{1}(\vec{\theta}) e^{i \psi_{1}} & \doteq \frac{i}{2} k e^{i \psi_{1}} \sin \theta_{1} \cos \theta_{2} \\
z_{2}=\frac{i}{2} k u_{2}(\vec{\theta}) e^{i \psi_{2}} & \doteq \frac{i}{2} k e^{i \psi_{2}} \sin \theta_{1} \sin \theta_{2} \cos \theta_{3} \\
& \cdots \\
z_{M-1}=\frac{i}{2} k u_{M-1}(\vec{\theta}) e^{i \psi_{M-1}} & \doteq \frac{i}{2} k e^{i \psi_{M-1}} \sin \theta_{1} \sin \theta_{2} \ldots \sin \theta_{M-1} \cos \theta_{M}, \\
z_{M}=\frac{i}{2} k u_{M}(\vec{\theta}) e^{i \psi_{M}} & \doteq \frac{i}{2} k e^{i \psi_{M}} \sin \theta_{1} \sin \theta_{2} \ldots \sin \theta_{M-1} \sin \theta_{M},
\end{aligned}
$$

where $k \in[0, \infty) ; \psi_{l} \in[0,2 \pi]$ for $l=0,1, \ldots, M$; and $\theta_{l} \in[0, \pi / 2]$ for $l=1,2, \ldots, M$, Eq. (2) can be rewritten as follows: 


$$
O=\int \mathrm{d} \mu[\vec{\psi}] \int \mathrm{d} \mu[\vec{\theta}] \int_{0}^{+\infty} \mathrm{d} k\left(\frac{k}{2}\right)^{2 M+1} \frac{1}{M !} \operatorname{Tr}\{O \exp [-i k X(\vec{\theta}, \vec{\psi})]\} \exp [i k X(\vec{\theta}, \vec{\psi})]
$$

Here we have used the notation

$$
\begin{gathered}
\int \mathrm{d} \mu[\vec{\psi}] \doteq \prod_{l=0}^{M} \int_{0}^{2 \pi} \frac{\mathrm{d} \psi_{l}}{2 \pi}, \quad \int \mathrm{d} \mu[\vec{\theta}] \doteq 2^{M} M ! \prod_{l=1}^{M} \int_{0}^{\pi / 2} d \theta_{l} \sin ^{2(M-l)+1} \theta_{l} \cos \theta_{l} \\
X(\vec{\theta}, \vec{\psi})=\frac{1}{2}\left[A^{\dagger}(\vec{\theta}, \vec{\psi})+A(\vec{\theta}, \vec{\psi})\right] \\
A(\vec{\theta}, \vec{\psi})=\sum_{l=0}^{M} e^{-i \psi_{l}} u_{l}(\vec{\theta}) a_{l}
\end{gathered}
$$

Notice that, thanks to the parameterization in Eq. (3), where $\sum_{l=0}^{+\infty} u_{l}^{2}(\vec{\theta})=1$, one has the commutation relation $\left[A(\vec{\theta}, \vec{\psi}), A^{\dagger}(\vec{\theta}, \vec{\psi})\right]=1$, which implies that $A(\vec{\theta}, \vec{\psi})$ and $A^{\dagger}(\vec{\theta}, \vec{\psi})$ themselves are annihilation and creation operators, respectively, of a bosonic mode. Also, by scanning all values of $\theta_{l} \in[0, \pi / 2]$ and $\psi_{l} \in[0,2 \pi]$, all possible linear combinations of the modes described by annihilation operators $a_{l}$, with $l=0, \ldots, M$, are obtained.

For a single mode of the radiation field the experimental homodyne probability distribution of a field quadrature with quantum efficiency $\eta<1$ is a Gaussian convolution with variance $\Delta_{\eta}^{2}=\frac{1-\eta}{4 \eta}$ of the ideal probability distribution. Therefore, for the quadrature operator $X(\vec{\theta}, \vec{\psi})$ in Eq. (6), one has the following identity for the moments generating function

$$
\left\langle e^{i k X}\right\rangle=\exp \left(\frac{1-\eta}{8 \eta} k^{2}\right) \int_{-\infty}^{+\infty} \mathrm{d} x e^{i k x} p_{\eta}(x ; \vec{\theta}, \vec{\psi})
$$

where $p_{\eta}(x ; \vec{\theta}, \vec{\psi})$ denotes the homodyne probability distribution of the quadrature $X(\vec{\theta}, \vec{\psi})$ with quantum efficiency $\eta$. Generally, $\eta$ can depend on the mode itself, i.e., it is a function $\eta=\eta(\vec{\theta}, \vec{\psi})$ of the selected mode. In the following, for simplicity, we assume $\eta$ to be mode independent, however. By taking the ensemble average on each side of Eq. (田) and using Eq. (8) one has

$$
\langle O\rangle=\int \mathrm{d} \mu[\vec{\psi}] \int \mathrm{d} \mu[\vec{\theta}] \int_{-\infty}^{+\infty} \mathrm{d} x p_{\eta}(x ; \vec{\theta}, \vec{\psi}) \mathcal{E}_{\eta}[O](x ; \vec{\theta}, \vec{\psi})
$$

where, for a given operator $O$, the function $\mathcal{E}_{\eta}[O](x ; \vec{\theta}, \vec{\psi})$ of $x, \vec{\theta}, \vec{\psi}$ has the following analytic expression

$$
\mathcal{E}_{\eta}[O](x ; \vec{\theta}, \vec{\psi})=\frac{\kappa^{M+1}}{M !} \int_{0}^{+\infty} \mathrm{d} t e^{-t+2 i \sqrt{\kappa t} x} t^{M} \operatorname{Tr}\{O: \exp [-2 i \sqrt{\kappa t} X(\vec{\theta}, \vec{\psi})]:\}
$$

with

$$
\kappa=\frac{2 \eta}{2 \eta-1}
$$


Equation (10) is the central result of this paper. For any given operator $O$ it provides the "unbiased estimator" to be averaged over all homodyne outcomes of the quadrature $X(\vec{\theta}, \vec{\psi})$ in order to obtain the ensemble average $\langle O\rangle$ for any unknown state of the radiation field. The homodyne outcomes for $X(\vec{\theta}, \vec{\psi})$ can be obtained by using a single LO that is prepared in the multimode coherent state $\otimes_{l=0}^{M}\left|\gamma_{l}\right\rangle$ with $\gamma_{l}=e^{i \psi_{l}} u_{l}(\theta) K / 2$ and $K \gg 1$. In fact, in this case the rescaled zero-frequency photocurrent at the output of a balanced homodyne detector is given by

$$
i=\sum_{l=0}^{M}\left(\gamma_{l}^{*} a_{l}+\gamma_{l} a_{l}^{\dagger}\right) / K
$$

which corresponds to the operator $X(\vec{\theta}, \vec{\psi})$. In the limit of a strong $\operatorname{LO}(K \rightarrow \infty)$, all moments of the current $i$ correspond to the moments of $X(\vec{\theta}, \vec{\psi})$, and the exact measurement of $X(\vec{\theta}, \vec{\psi})$ is then realized. Notice that for modes $a_{l}$ with different frequencies, in the d.c. photocurrent in Eq. (12) each LO with amplitude $\gamma_{l}$ selects the mode $a_{l}$ at the same frequency (and polarization). For the effect of less-than-unity quantum efficiency, previous considerations on Eq. (8) apply.

In order to obtain the ensemble average in Eq. (9) as an experimental average over the homodyne outcomes, one needs to satisfy the validity conditions of the central-limit theorem. Since in the strong LO approximation the probability $p_{\eta}(x ; \vec{\theta}, \vec{\psi})$ must decay as a Gaussian for large $x$, it follows that the integral in Eq. (9) can be experimentally sampled for any a priori unknown probability distribution $p_{\eta}(x ; \vec{\theta}, \vec{\psi})$ if $\mathcal{E}_{\eta}[O](x ; \vec{\theta}, \vec{\psi})$ increases slower than $\exp \left(\kappa x^{2}\right)$ for large $x$, and is bounded for $|x|<+\infty$. In this case one is guaranteed that the integral in Eq. (9) can be statistically sampled over a sufficiently large set of data. The average values for different experiments will be Gaussian distributed around the mathematical expectation in Eq. (9), allowing estimation of the confidence intervals, which will decrease as the inverse square-root of the number of experimental data. In general, the boundedness of $\mathcal{E}_{\eta}[O](x ; \vec{\theta}, \vec{\psi})$ for $|x|<+\infty$ will pose lower bounds for the quantum efficiency $\eta$ below which the measurement cannot be performed, similarly to what happens in the one-mode case [12]. This limitation is due to the generality of the method, which is perfectly unbiased, and makes no a priori assumption on the state of the radiation field, the only approximation being that of a strong LO. This should be contrasted with other methods, as the maximum entropy method [13] or the maximum likelihood method [14, 15], which do not suffer such limitation on the quantum efficiency; however they are generally biased and based on assumptions for the state of the radiation field.

Equation (9) can be specialized to some observables $O$ of interest. In particular, one can estimate the matrix element $\left\langle\left\{n_{l}\right\}|R|\left\{m_{l}\right\}\right\rangle$ of the multimode density operator $R$. This will be obtained by averaging the following estimator:

$$
\begin{aligned}
\mathcal{E}_{\eta}\left[\left|\left\{m_{l}\right\}\right\rangle\left\langle\left\{n_{l}\right\}\right|\right](x ; \vec{\theta}, \vec{\psi}) & =e^{-i \sum_{l=0}^{M}\left(n_{l}-m_{l}\right) \psi_{l}} \frac{\kappa^{M+1}}{M !} \prod_{l=0}^{M}\left\{\left[-i \sqrt{\kappa} u_{l}(\vec{\theta})\right]^{\mu_{l}-\nu_{l}} \sqrt{\frac{\nu_{l} !}{\mu_{l} !}}\right\} \\
& \times \int_{0}^{+\infty} \mathrm{d} t e^{-t+2 i \sqrt{\kappa t} x} t^{M+\sum_{l=0}^{M}\left(\mu_{l}-\nu_{l}\right) / 2} \prod_{l=0}^{M} L_{\nu_{l}}^{\mu_{l}-\nu_{l}}\left[\kappa u_{l}^{2}(\vec{\theta}) t\right]
\end{aligned}
$$


where $\mu_{l}=\max \left(m_{l}, n_{l}\right), \nu_{l}=\min \left(m_{l}, n_{l}\right)$, and $L_{n}^{\alpha}(z)$ denotes the customary generalized Laguerre polynomial of variable z. For diagonal matrix elements, Eq. (13) simplifies to

$$
\mathcal{E}_{\eta}\left[\left|\left\{n_{l}\right\}\right\rangle\left\langle\left\{n_{l}\right\}\right|\right](x ; \vec{\theta}, \vec{\psi})=\frac{\kappa^{M+1}}{M !} \int_{0}^{+\infty} \mathrm{d} t e^{-t+2 i \sqrt{\kappa t} x} t^{M} \prod_{l=0}^{M} L_{n_{l}}\left[\kappa u_{l}^{2}(\vec{\theta}) t\right]
$$

with $L_{n}(z)$ denoting the customary Laguerre polynomial in $z$. Notice that the estimator in Eq. (14) does not depend on the phases $\psi_{l}$; only the knowledge of the angles $\theta_{l}$ is needed. Using the following identity for the Laguerre polynomials [16]:

$$
L_{n}^{\alpha_{0}+\alpha_{1}+\ldots+\alpha_{M}+M}\left(x_{0}+x_{1}+\ldots+x_{M}\right)=\sum_{i_{0}+i_{1}+\ldots+i_{M}=n} L_{i_{0}}^{\alpha_{0}}\left(x_{0}\right) L_{i_{1}}^{\alpha_{1}}\left(x_{1}\right) \ldots L_{i_{M}}^{\alpha_{M}}\left(x_{M}\right),
$$

from Eq. (14) one can easily derive the estimator for the probability distribution of the total number of photons $N=\sum_{l=0}^{M} a_{l}^{\dagger} a_{l}$

$$
\mathcal{E}_{\eta}[|n\rangle\langle n|](x ; \vec{\theta}, \vec{\psi})=\frac{\kappa^{M+1}}{M !} \int_{0}^{+\infty} \mathrm{d} t e^{-t+2 i \sqrt{\kappa t} x} t^{M} L_{n}^{M}[\kappa t]
$$

where $|n\rangle$ denotes the eigenvector of $N$ with eigenvalue $n$. Notice that the estimator in Eq. (16) does not depend on any of the phases $\psi_{l}$ or the angles $\theta_{l}$, and thus their knowledge is not needed in the measurement of the probability distribution of the total number of photons.

Now we specialize to the case of only two modes (i.e., $M=1$ and $\vec{\theta}$ is a scalar $\theta$ ). The joint photon-number probability distribution is obtained by averaging the following estimator:

$$
\mathcal{E}_{\eta}[|n, m\rangle\langle n, m|]\left(x ; \theta, \psi_{0}, \psi_{1}\right)=\kappa^{2} \int_{0}^{+\infty} \mathrm{d} t e^{-t+2 i \sqrt{\kappa t} x} t L_{n}\left(\kappa t \cos ^{2} \theta\right) L_{m}\left(\kappa t \sin ^{2} \theta\right) .
$$

Analogously, using Eq. (10) one can derive the following estimator for the four dimensional Q-function:

$$
\begin{aligned}
& \mathcal{E}_{\eta}[|\alpha, \beta\rangle\langle\alpha, \beta|]\left(x ; \theta, \psi_{0}, \psi_{1}\right)= \\
& \kappa^{2} \Phi\left(2, \frac{1}{2} ;-\kappa\left[x-\frac{1}{2} \cos \theta \operatorname{Im}\left(\alpha^{*} e^{i \psi_{0}}\right)-\frac{1}{2} \sin \theta \operatorname{Im}\left(\beta^{*} e^{i \psi_{1}}\right)\right]^{2}\right)
\end{aligned}
$$

where $|\alpha, \beta\rangle$ with $\alpha, \beta \in \mathbb{C}$ denotes a two-mode coherent state, and $\Phi(a, b ; z)$ is the customary confluent hypergeometric function of $z$. The estimator (16) for the probability distribution of the total number of photons can be written as

$$
\mathcal{E}_{\eta}[|n\rangle\langle n|]\left(x ; \theta, \psi_{0}, \psi_{1}\right)=\kappa^{2} \int_{0}^{+\infty} \mathrm{d} t e^{-t+2 i \sqrt{\kappa t} x} t L_{n}^{1}[\kappa t] .
$$

For the total number of photons one can also derive the estimator for the moment generating function, using the generating function for the Laguerre polynomials [16]. One obtains

$$
\mathcal{E}_{\eta}\left[z^{a^{\dagger} a+b^{\dagger} b}\right]\left(x ; \theta, \psi_{0}, \psi_{1}\right)=\frac{1}{\left(z+\frac{1-z}{\kappa}\right)^{2}} \Phi\left(2, \frac{1}{2} ;-\frac{1-z}{z+\frac{1-z}{\kappa}} x^{2}\right)
$$


where we have denoted by $a$ and $b$ the annihilation operators of the two modes. For the first two moments one obtains the simple expressions

$$
\begin{aligned}
\mathcal{E}_{\eta}\left[a^{\dagger} a+b^{\dagger} b\right]\left(x ; \theta, \psi_{0}, \psi_{1}\right) & =4 x^{2}+\frac{2}{\kappa}-2, \\
\mathcal{E}_{\eta}\left[\left(a^{\dagger} a+b^{\dagger} b\right)^{2}\right]\left(x ; \theta, \psi_{0}, \psi_{1}\right) & =8 x^{4}+\left(\frac{24}{\gamma}-20\right) x^{2}+\frac{6}{\gamma^{2}}-\frac{10}{\gamma}+4 .
\end{aligned}
$$

It is worth noting that analogous estimators for the photon-number difference between the two modes are singular and one needs a cutoff procedure, similar to the one used in Ref. 10 for recovering the correlation between the modes by means of the customary two-mode tomography. The singular behavior of the estimators for the photon-difference operators can be understood simply from the fact that to extract information pertaining to a single mode only one needs a delta-function at $\theta=0$ for mode $a$, or $\theta=\pi / 2$ for mode $b$, and, in this case, one could better use the original one-mode tomography method [12 by setting the LO to the proper mode of interest.

Finally, we note that for the case of two-mode tomography the estimator $\mathcal{E}_{\eta}$ can be averaged by taking the integral

$$
\langle O\rangle=\int_{0}^{2 \pi} \frac{\mathrm{d} \psi_{0}}{2 \pi} \int_{0}^{2 \pi} \frac{\mathrm{d} \psi_{1}}{2 \pi} \int_{-1}^{1} \frac{\mathrm{d}(\cos 2 \theta)}{2} \int_{-\infty}^{+\infty} \mathrm{d} x p_{\eta}\left(x ; \theta, \psi_{0}, \psi_{1}\right) \mathcal{E}_{\eta}[O]\left(x ; \theta, \psi_{0}, \psi_{1}\right)
$$

over the random parameters $\cos (2 \theta), \psi_{0}$, and $\psi_{1}$. For example, in the case of two radiation modes having the same frequency but orthogonal polarizations, $\theta$ represents a random rotation of the polarizations, whereas $\psi_{0}$ and $\psi_{1}$ denote the relative phases between the LO and the two modes, respectively.

\section{NUMERICAL RESULTS FOR TWO MODES}

In this section we present some Monte-Carlo simulations in order to estimate the working experimental conditions for performing the single-LO tomography on two-mode fields. We focus our attention on the twin-beam state, usually generated by spontaneous parametric downconversion, namely

$$
|\Psi\rangle=\left(1-|\xi|^{2}\right)^{\frac{1}{2}} \sum_{n=0}^{\infty} \xi^{n}|n\rangle_{a}|n\rangle_{b}
$$

For the simulations we use the following homodyne probability distribution that is derived in the Appendix:

$$
p_{\eta}\left(x ; \theta, \psi_{0}, \psi_{1}\right)=\frac{1}{\sqrt{2 \pi \Delta_{\eta}^{2}\left(\theta, \psi_{0}, \psi_{1}\right)}} \exp \left(-\frac{x^{2}}{2 \Delta_{\eta}^{2}\left(\theta, \psi_{0}, \psi_{1}\right)}\right)
$$

where the variance $\Delta_{\eta}^{2}\left(\theta, \psi_{0}, \psi_{1}\right)$ is given by

$$
\Delta_{\eta}^{2}\left(\theta, \psi_{0}, \psi_{1}\right)=\frac{1+|\xi|^{2}+2|\xi| \sin 2 \theta \cos \left(\psi_{0}+\psi_{1}-\arg \xi\right)}{4\left(1-|\xi|^{2}\right)}+\frac{1-\eta}{4 \eta}
$$


In the case of two radiation modes having the same frequency but orthogonal polarizations, Eq. (25) gives the theoretical probability of outcome $x$ for the homodyne measurement at a polarization angle $\theta, \psi_{0}$ and $\psi_{1}$ denoting the relative phases between the $\mathrm{LO}$ and the two modes, respectively.

We study the tomographic measurement of the joint photon-number probability distribution and the probability distribution for the total number of photons with use of the estimators in Eqs. (17) and (19), respectively. Moreover, we reconstruct the matrix elements

$$
C_{n, m} \equiv{ }_{a}\left\langle\left. m\right|_{b}\langle m \mid \Psi\rangle\langle\Psi \mid n\rangle_{a} \mid n\right\rangle_{b}
$$

that reveal the coherence of the twin-beam state by using the estimator in Eq. (13). For the twin-beam state in Eq. (24), one should have

$$
C_{n, m}=\left(1-|\xi|^{2}\right) \xi^{m} \xi^{* n} .
$$

The estimators have been numerically evaluated by applying the Gauss method for calculating the integral in Eq. (13), which results in a fast and sufficiently precise algorithm with use of just 150 evaluation points. Notice that in the present case there is no convenience in using the factorization formula given in Ref. [9], as in that case an integral of a product of functions is needed.

In Fig. 1 a Monte-Carlo simulation of the joint photon-number probability distribution is presented. The simulated values compare very well with the theoretical ones. We have done a careful analysis of the statistical errors for various twin-beam states by constructing histograms of deviations of the results from different simulated experiments from the theoretical ones. In comparison to the customary two-LO tomography [10], where for $\eta=1$ the statistical errors saturate for increasingly large $n$ and $m$, here we have statistical errors that are slowly increasing versus $n$ and $m$. This is due to the fact that the range of the estimator in Eq. (17) increases versus $n$ and $m$. Overall we find that for any given quantum efficiency the statistical errors are generally slightly larger than those obtained with the two-LO method. The convenience of using a single LO then comes with its own price tag.

By using the estimator in Eq. (19) we have also constructed the probability distribution for the total number of photons $N$ of the twin-beam state with unity (Fig. 21) as well as less-than-unity (Fig. 31) quantum efficiencies. Notice the dramatic increase of error bars

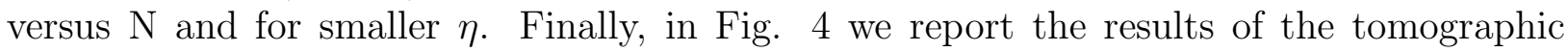
measurement of the matrix elements $C_{n, m}$ defined in Eq. (27). Because the reconstructed $C_{n, m}$ is close to the theoretically expected value in Eq. (28), these reveal the purity of the twin-beam state, which cannot be inferred from the thermal diagonal distribution of Fig. [1.

\section{AN APPLICATION TO THE GHZ STATE}

Multimode homodyne tomography allows one to verify the generation of multimode states that are of interest in studies of the foundations of quantum mechanics. An example is the Greenberger-Horne-Zeilinger (GHZ) state [17], which is a 6-mode state given by

$$
|\mathrm{GHZ}\rangle \equiv \frac{1}{\sqrt{2}}\left(\left|1 a_{o} 1 b_{o} 1 c_{o}\right\rangle-\left|1 a_{e} 1 b_{e} 1 c_{e}\right\rangle\right),
$$


wherein $o, e$ denote a couple of orthogonal polarizations; $a, b, c$ pertain to electromagnetic modes with different wavevectors and/or frequencies; and the notation $|1,1,1\rangle$ represents the tensor product of three single-photon Fock states. The GHZ state is very interesting as it leads to correlations between three particles that are in contradiction with the EinsteinPodolsky-Rosen idea of "elements of reality" [18]. We note here that no experiment has yet succeeded in realizing the GHZ state.

A tomographic measurement of the state in Eq. (29) can be suitably performed by varying randomly the phases and polarizations of the pairs of modes $a_{o, e}, b_{o, e}$, and $c_{o, e}$, and then collecting homodyne outcomes by using three different LO's. The need of using three separate LO's in the present case is that in the actual experimental arrangement [19] the three beams corresponding to modes $a_{o, e}, b_{o, e}$, and $c_{o, e}$ come with different wave-vectors and thus are spatially separated. Hence, such an experimental arrangement here gives the opportunity of using a combination of the present multimode method and the usual manyLO method based on the product of single-mode estimators.

A simple tomographic check of the GHZ-state production consists of measuring the expectation value on the projector $|\phi\rangle\langle\phi|$, where

$$
|\phi\rangle \equiv \frac{1}{\sqrt{2}}\left(\left|1 a_{o} 1 b_{o} 1 c_{o}\right\rangle+e^{i \phi}\left|1 a_{e} 1 b_{e} 1 c_{e}\right\rangle\right),
$$

and comparing the result with the theoretical value, namely,

$$
C(\phi) \equiv|\langle\phi \mid \mathrm{GHZ}\rangle|^{2}=\frac{1}{2}(1-\cos \phi) .
$$

Notice that for $\phi=\pi$ the function $C(\phi)$ represents the fidelity of the GHZ-state production. In addition, the same set of homodyne data allows one to recover the whole interference profile in Eq. (31) for all values of $\phi$.

In Fig. 5 we report the results of a Monte-Carlo simulation of the tomographic measurement of $C(\phi)$ in Eq. (31). We used $5 \times 10^{7}$ homodyne data samples and assumed a quantum efficiency $\eta=85 \%$. For these parameters, the simulated $C(\phi)$ compares very well with the theoretical one.

\section{CONCLUSIONS}

We have presented a generalization of the quantum homodyne tomography method to many modes of the radiation field that requires the use of only a single LO. By varying suitable random parameters the LO scans over all the linear combinations of the field modes. We have also provided a general method to obtain the "unbiased estimator" for a generic multimode operator. The quantum expectation value of such an operator can be evaluated for any unknown state of the radiation field by averaging the estimator over the homodyne outcomes that are collected by using a single LO. The estimators for some observables, such as the matrix elements of the multimode density operator and the total number of photons, have been explicitly evaluated. For the two-mode case we derived the estimator for the four-dimensional Q-function and the moments generating function of the total number of photons. By means of Monte-Carlo simulations we have analyzed in detail the case 
of the twin-beam state, namely the two-mode state produced by nondegenerate parametric amplification (spontaneous downconversion). For quantum efficiency of homodyne detection in the $80-90 \%$ range and with number of data samples of order $10^{6}-10^{7}$, the simulations show that measurements of the joint photon-number probability, the distribution of the total number of photons, and the density-matrix elements are experimentally feasible.

We have also shown an application of the method of multimode homodyne tomography to the measurement of the radiation field prepared in the Greenberger-Horne-Zeilinger state. The results of our simulations suggest that with a number of homodyne data samples around $10^{7}$ and a homodyne detection efficiency of $85 \%$ the method would allow the reconstruction of such an interesting state of the radiation field with relatively small statistical errors.

\section{APPENDIX}

In this appendix we derive the theoretical probability distribution $p_{\eta}\left(x ; \theta, \psi_{0}, \psi_{1}\right)$ of the twin-beam state

$$
|\Psi\rangle=S(\chi)|0\rangle_{a}|0\rangle_{b}=\left(1-|\xi|^{2}\right)^{\frac{1}{2}} \sum_{n=0}^{\infty} \xi^{n}|n\rangle_{a}|n\rangle_{b},
$$

where $S(\chi)=\exp \left(\chi a^{\dagger} b^{\dagger}-\chi^{*} a b\right)$ and $\xi=e^{i \arg \chi} \tanh |\chi|$. For unity quantum efficiency, the probability density $p\left(x ; \theta, \psi_{0}, \psi_{1}\right)$ is defined as follows:

$$
\begin{aligned}
p\left(x ; \theta, \psi_{0}, \psi_{1}\right) & =\operatorname{Tr}\left[U^{\dagger}|x\rangle_{a a}\left\langle x\left|\otimes 1_{b} U\right| \Psi\right\rangle\langle\Psi|\right] \\
& ={ }_{a}\left\langle\left. 0\right|_{b}\left\langle 0\left|S^{\dagger}(\chi) U^{\dagger}\right| x\right\rangle_{a a}\left\langle x\left|\otimes 1_{b} U S(\chi)\right| 0\right\rangle_{a} \mid 0\right\rangle_{b},
\end{aligned}
$$

where $|x\rangle_{a}$ is the eigenvector of the quadrature $x=\frac{1}{2}\left(a^{\dagger}+a\right)$ with eigenvalue $x$ and $U$ is the unitary operator achieving the mode transformation

$$
U^{\dagger}\left(\begin{array}{l}
a \\
b
\end{array}\right) U=\left(\begin{array}{cc}
e^{-i \psi_{0}} \cos \theta & e^{-i \psi_{1}} \sin \theta \\
-e^{i \psi_{1}} \sin \theta & e^{i \psi_{0}} \cos \theta
\end{array}\right)\left(\begin{array}{l}
a \\
b
\end{array}\right)
$$

In the case of two radiation modes having the same frequency but orthogonal polarizationsthe case of Type II phase-matched parametric amplifier-Eq. (25) gives the theoretical probability of outcome $x$ for the homodyne measurement at a polarization angle $\theta$ with respect to the polarization of the $a$ mode, and with $\psi_{0}$ and $\psi_{1}$ denoting the relative phases between the LO and the two modes, respectively. By using the Dirac- $\delta$ representation of the $X$-quadrature projector

$$
|x\rangle\langle x|=\int_{-\infty}^{+\infty} \frac{\mathrm{d} \lambda}{2 \pi} \exp [i \lambda(X-x)],
$$

Eq. (33) can be rewritten as follows:

$$
\begin{aligned}
& p\left(x ; \theta, \psi_{0}, \psi_{1}\right)=\int_{-\infty}^{+\infty} \frac{\mathrm{d} \lambda}{2 \pi}{ }_{a}\left\langle 0\left|{ }_{b}\left\langle 0\left|S^{\dagger}(\chi) U^{\dagger} e^{i \lambda\left(X_{a}-x\right)} U S(\chi)\right| 0\right\rangle_{a}\right| 0\right\rangle_{b}=\int_{-\infty}^{+\infty} \frac{\mathrm{d} \lambda}{2 \pi} e^{-i \lambda x} \times \\
& { }_{a}\left\langle\left. 0\right|_{b}\langle 0| \exp \left\{i \frac{\lambda}{2}\left[\left(e^{-i \psi_{0}} \mu \cos \theta+e^{i \psi_{1}} \nu^{*} \sin \theta\right) a+\left(e^{i \psi_{0}} \nu^{*} \cos \theta+e^{-i \psi_{1}} \mu \sin \theta\right) b+\text { H.c. }\right]\right\} \mid 0\right\rangle_{a}|0\rangle_{b},
\end{aligned}
$$


where we have used Eq. (34) and the transformation

$$
S^{\dagger}(\chi)\left(\begin{array}{c}
a \\
b^{\dagger}
\end{array}\right) S(\chi)=\left(\begin{array}{cc}
\mu & \nu \\
\nu^{*} & \mu
\end{array}\right)\left(\begin{array}{c}
a \\
b^{\dagger}
\end{array}\right)
$$

with $\mu=\cosh |\chi|$ and $\nu=e^{i \arg \chi} \sinh |\chi|$. Upon defining

$$
\begin{aligned}
& K C=e^{-i \psi_{0}} \mu \cos \theta+e^{i \psi_{1}} \nu^{*} \sin \theta, \\
& K D=e^{i \psi_{0}} \nu^{*} \cos \theta+e^{-i \psi_{1}} \mu \sin \theta,
\end{aligned}
$$

where $K \in \mathbb{R}$ and $C, D \in \mathbb{C}$, with $|C|^{2}+|D|^{2}=1$ one has

$$
K^{2}=\mu^{2}+|\nu|^{2}+2 \mu|\nu| \sin 2 \theta \cos \left(\psi_{0}+\psi_{1}-\arg \nu\right) \text {. }
$$

Now, since the unitary transformation

$$
\left(\begin{array}{cc}
C & D \\
D^{*} & C^{*}
\end{array}\right)\left(\begin{array}{l}
a \\
b
\end{array}\right) \longrightarrow\left(\begin{array}{l}
a \\
b
\end{array}\right)
$$

has no effect on the vacuum state, Eq. (36) leads to the following Gaussian distribution:

$$
\begin{aligned}
& p\left(x ; \theta, \psi_{0}, \psi_{1}\right)=\int_{-\infty}^{+\infty} \frac{\mathrm{d} \lambda}{2 \pi} e^{-i \lambda x}{ }_{a}\langle 0|{ }_{b}\langle 0| \exp \left\{i K \frac{\lambda}{2}[(C a+D b)+\text { H.c. }]\right\}|0\rangle_{a}|0\rangle_{b} \\
& =\int_{-\infty}^{+\infty} \frac{\mathrm{d} \lambda}{2 \pi} e^{-i \lambda x}{ }_{a}\left\langle 0\left|\exp \left\{i K \frac{\lambda}{2}\left[a+a^{\dagger}\right]\right\}\right| 0\right\rangle_{a}=\left.\left.\frac{1}{K}\right|_{a}\langle 0 \mid x / K\rangle_{a}\right|^{2} \\
& =\frac{1}{\sqrt{2 \pi \Delta^{2}\left(\theta, \psi_{0}, \psi_{1}\right)}} \exp \left(-\frac{x^{2}}{2 \Delta^{2}\left(\theta, \psi_{0}, \psi_{1}\right)}\right),
\end{aligned}
$$

where the variance $\Delta^{2}\left(\theta, \psi_{0}, \psi_{1}\right)$ is given by

$$
\Delta^{2}\left(\theta, \psi_{0}, \psi_{1}\right)=\frac{K^{2}}{4}=\frac{1+|\xi|^{2}+2|\xi| \sin 2 \theta \cos \left(\psi_{0}+\psi_{1}-\arg \xi\right)}{4\left(1-|\xi|^{2}\right)}
$$

Taking into account the Gaussian convolution that results from less-than-unity quantum efficiency, the variance just increases as $\Delta^{2}\left(\theta, \psi_{0}, \psi_{1}\right) \rightarrow \Delta_{\eta}^{2}\left(\theta, \psi_{0}, \psi_{1}\right)=\Delta^{2}\left(\theta, \psi_{0}, \psi_{1}\right)+\frac{1-\eta}{4 \eta}$. Notice that the probability distribution in Eq. (41) corresponds to a squeezed vacuum for $\theta=\frac{\pi}{4}$ and $\psi_{0}+\psi_{1}-\arg \xi=0$ or $\pi$.

\section{ACKNOWLEDGEMENTS}

This work has been supported by the INFM project PRA-CAT 1997 and by the MURST Cofinanziamento program "Amplificazione e Rivelazione di Radiazione Quantistica". The research of P.K. is partially supported by the U.S. Office of Naval Research. 


\section{REFERENCES}

[1] D. T. Smithey, M. Beck, M. G. Raymer and A. Faridani, Phys. Rev. Lett. 70, 1244 (1993).

[2] M. Munroe, D. Boggavarapu, M. E. Anderson, and M. G. Raymer, Phys. Rev. A 52, R924 (1995).

[3] G. Breitenbach, S. Schiller and J. Mlynek, Nature 387, 471 (1997).

[4] G. M. D'Ariano, "Quantum estimation theory and optical detection," in Quantum Optics and the Spectroscopy of Solids, T. Hakioğlu and A. S. Shumovsky, Eds. (Kluwer, Dordrecht 1997) p. 139.

[5] U. Janicke and M. Wilkens, J. Mod. Opt. 42, 2183, (1995); S. Wallentowitz, W. Vogel, Phys. Rev. Lett. 75, 2932 (1995); S. H. Kienle, M. Freiberger, W. P. Schleich, and M. G. Raymer, in Experimental Methaphisics: Quantum Mechanical Studies for Abner Shimony, ed. S. Cohen et al. (Kluwer, Lancaster 1997), p. 121; C. Kurtsiefer, T. Pfau, and J. Mlynek, Nature 386, 150 (1997).

[6] T. J. Dunn, I. A. Walmsley, and S. Mukamel, Phys. Rev. Lett. 74, 884 (1995).

[7] D. Leibfried, D. M. Meekhof, B. E. King, C. Monroe, W. M. Itano, and D. J.Wineland, Phys. Rev. Lett. 77, 4281 (1996).

[8] G. M. D'Ariano, in "Quantum Communication, Computing, and Measurement", ed. by O. Hirota, A. S. Holevo and C. M. Caves, Plenum Publishing (New York and London 1997), p. 253.

[9] M. G. Raymer, D. F. McAlister, and U. Leonhardt, Phys. Rev. A 54, 2397 (1996); D. F. McAlister and M. G. Raymer, Phys. Rev. A 55, R1609 (1997).

[10] G. M. D'Ariano, M. Vasilyev, and P. Kumar, Phys. Rev. A 58636 (1998).

[11] J. H. Shapiro, A. Shakeel, JOSA B 14, 232 (1997); D. Levandovsky, PhD Proposal, Northwestern University, 1996 (unpublished).

[12] G. M. D'Ariano, U. Leonhardt and H. Paul, Phys. Rev. A 52, R1801 (1995).

[13] V. Bužek, G. Adam, and G. Drobný, Ann. Phys. (N.Y.) 245, 37 (1996).

[14] K. Banaszek, Phys. Rev. A 575013 (1998).

[15] Z. Hradil, Phys. Rev. A 55 R1561 (1997).

[16] I.S. Gradstein and I.M. Ryzhik: Table of Integrals, Series, and Products (Academic Press, New York, 1980).

[17] D. M. Greenberger, M. A. Horne, and A. Zeilinger, in Bell's Theorem, Quantum Theory, and Conceptions of the Universe, M. Kafatos, Ed. (Kluwer, Dordrecht 1989) p. 69.

[18] A. Einstein, B. Podolsky, and N. Rosen, Phys. Rev. 47, 777 (1935).

[19] T. E. Keller, M. H. Rubin, Y. H. Shih, and L. Wu, Phys. Rev. A 57, 2076 (1998), and references therein. 

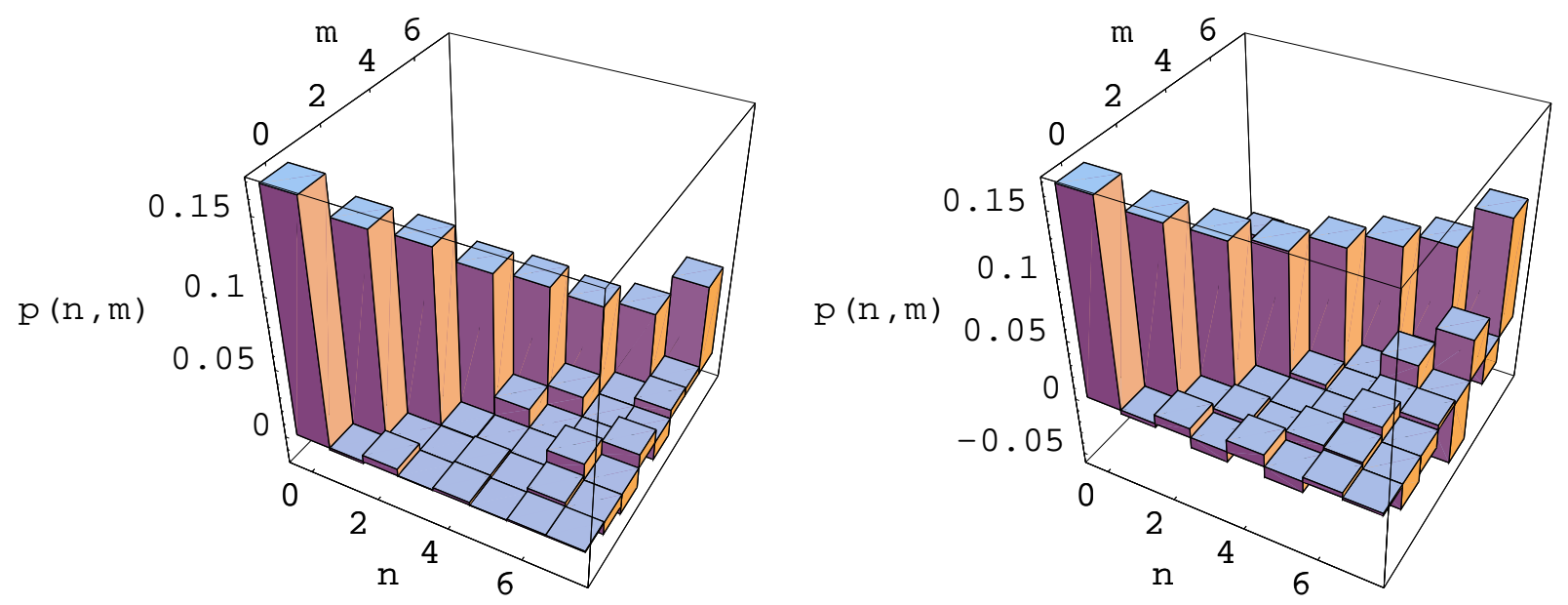

FIG. 1. Two-mode photon-number probability $p(n, m)$ of the twin-beam state of parametric fluorescence in Eq. (24) for average number of photons per beam $\bar{n}=|\xi|^{2} /\left(1-|\xi|^{2}\right)=5$ obtained by a Monte-Carlo simulation of the probability in Eqs. (25) and (26) with random parameters $\cos 2 \theta, \psi_{0}$, and $\psi_{1}$, using the estimator in Eq. (17). On the left we have quantum efficiency $\eta=1$ and $10^{6}$ data samples were used in the reconstruction. On the right quantum efficiency $\eta=0.9$, and $5 \times 10^{6}$ data samples were used. The theoretical values of off-diagonal $p(n, m)$ are zero; for a comparison between theoretical and experimental diagonal $p(n, n)$ probabilities and their relative statistical errors, see analogous experiments in Figs. 2 and 3 . 


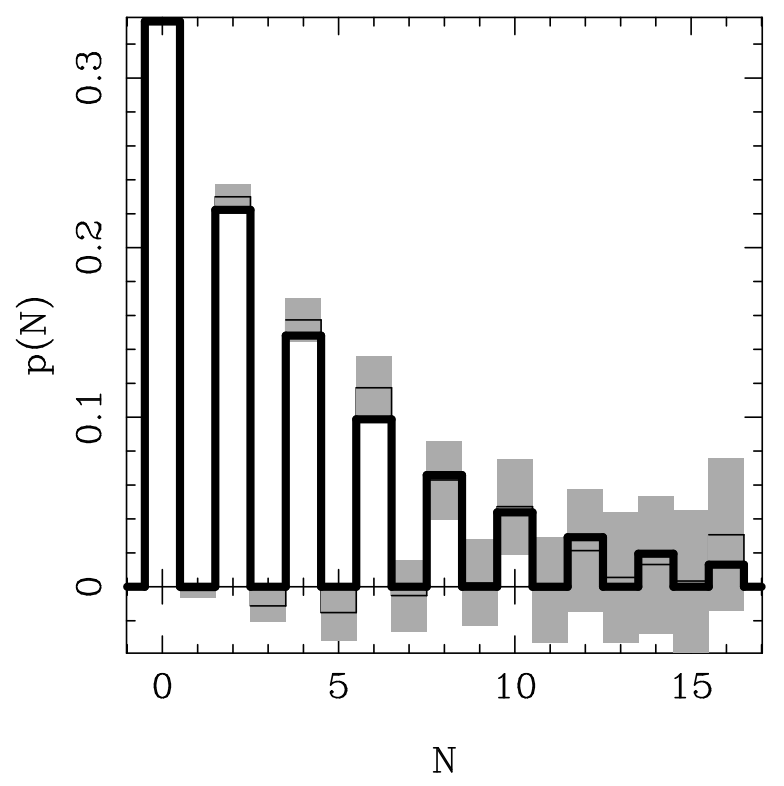

FIG. 2. Probability distribution for the total number of photons of the twin-beam state in Eq. (24) for average number of photons per beam $\bar{n}=2$ obtained using the estimator in Eq. (19). The oscillation of the total photon-number probability due to the perfect correlation of the twin-beam state has been reconstructed by simulating $10^{6}$ data samples with quantum efficiency $\eta=1$. The theoretical probability (thick solid line) is superimposed onto the result of the Monte-Carlo experiment; the latter is shown by the thin solid line with the statistical errors in gray shade. 

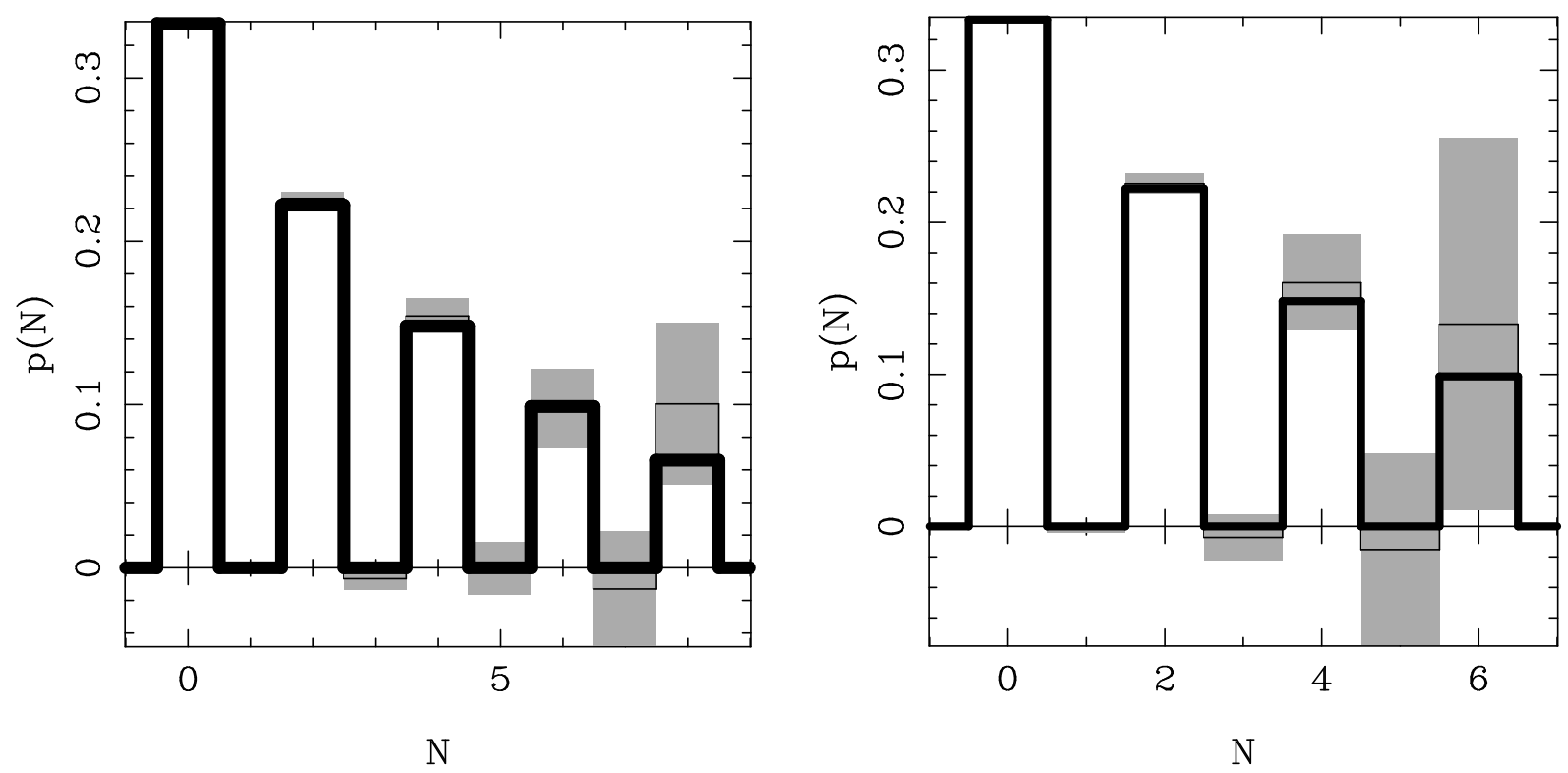

FIG. 3. Similar to Fig. 2, but for quantum efficiency $\eta=0.9$ and $10^{7}$ data samples (on the left), and $\eta=0.8$ and $2 \times 10^{7}$ data samples (on the right). Notice the dramatic increase of error bars (in gray shade) versus $\mathrm{N}$ and for smaller $\eta$. 

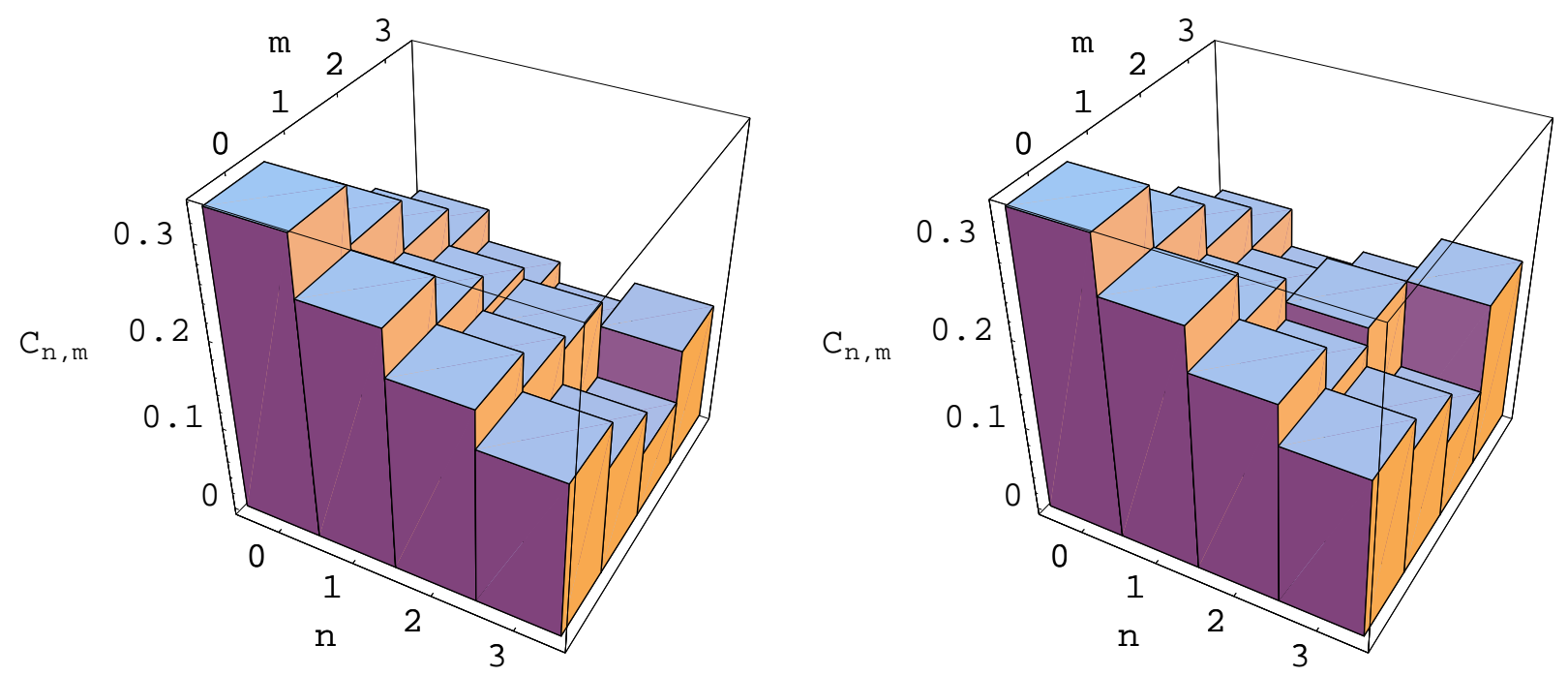

FIG. 4. Tomographic reconstruction of the matrix elements $C_{n, m} \equiv{ }_{a}\left\langle\left. m\right|_{b}\langle m \mid \Psi\rangle\langle\Psi \mid n\rangle_{a} \mid n\right\rangle_{b}$ of the twin-beam state of parametric fluorescence in Eq. (24) for average number of photons per beam $\bar{n}=2$, obtained using the estimator in Eq. (13). On the left we used $10^{6}$ simulated data samples and quantum efficiency $\eta=0.9$; on the right $3 \times 10^{6}$ data samples and $\eta=0.8$. The coherence of the twin-beam state is easily recognized as $C_{n, m}$ varies little for $n+m=$ constant [ $\xi$ in Eq. (24) has been chosen real]. For a typical comparison between theoretical and experimental matrix elements and their relative statistical errors, see experiments in Figs. 2 and 3. 


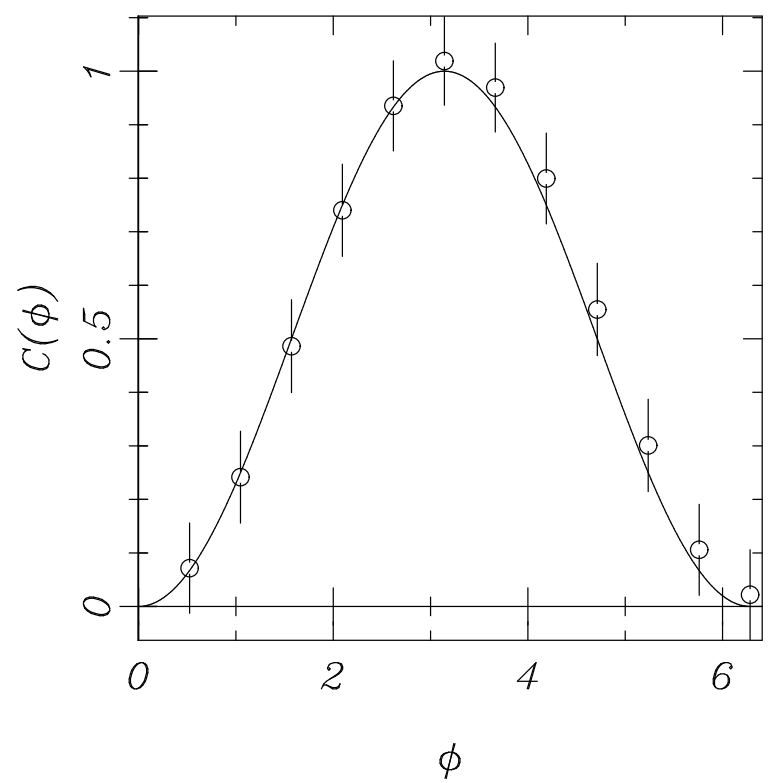

FIG. 5. Tomographic measurement of the overlap $C(\phi)$ between the GHZ state in Eq. (29) and the state $|\phi\rangle$ in Eq. (30) with varying phase $\phi$. The value for $\phi=\pi$ represents the fidelity between the experimental state and the theoretical one. Here a Monte-Carlo simulation with $N=2.5 \times 10^{7}$ data samples and quantum efficiency $\eta=0.85$. The bars represent the statistical error, whereas the solid line is the theoretical value of $C(\phi)$. All points are obtained from the same data samples (which causes the evident correlation between the statistical deviations). 